\title{
CDISC SEND Challenge Agent Category Response Terminology
}

National Cancer Institute

\section{Source}

National Cancer Institute. CDISC SEND Challenge Agent Category Response

Terminology. NCI Thesaurus. Code C158120.

Terminology associated with the challenge agent category Response codelist of the Clinical Data Interchange Standards Consortium (CDISC) Standard for the Exchange of Non-clinical Data (SEND). 